\title{
Antimicrobial prophylaxis in patients after hematopoietic cell transplantation: results of a survey of the Polish Federation of Bone Marrow Transplant Centers
}

(๑) 2020 Polish Society of Hematology and Transfusion Medicine, Insitute of Hematology and Transfusion Medicine. Published by Sciendo. All rights reserved.

\section{Introduction}

Infection is a major cause of morbidity and one of the major causes of death after hematopoietic cell transplantation (HCT). Since infection may compromise the benefit of transplantation, each infectious complication remains a clinical challenge in patients after transplantation. In European Group for Blood and Marrow Transplantation (EBMT) analysis of 114,491 patients with 5-year follow-up, a total of 55,668 patients were reported dead [1]. In $22.3 \%$ of all deaths, the cause was infection. The rate of deaths from infections varied over calendar time and posttransplant phase. Overall the rate of infectious deaths decreased in all phases after autologous HCT (auto-HCT), and up to day 100 and beyond after allogeneic HCT (allo-HCT) which was the second cause of death after auto-HCT and third after allo-HCT.

With respect to the risk of infectious complications, posttransplant period is usually divided into the following intervals: very early (up to day 30 and beyond), early (between days 30 and 100), late (between day 100 and 1 year), and very late (beyond 1 year). Alternatively, the phases can be divided into pretransplant phase, neutropenic phase, and immunosuppression phase; in allo-HCT setting, additional phase of immunosuppressive prophylaxis and graft-versus-host disease (GVHD) is distinguished. The risk of infections is particularly high during very early phase due to the toxicity of conditioning and neutropenia, as well as immunosuppressive therapy in case of allo-HCT; additionally, immunosuppression can be continued for many months after hematological recovery, especially in patients diagnosed with GVHD. Incidence of infections is particularly high during very early and early posttransplant phase [2-6]; however, the patient remains at risk of infectious complications from the beginning of conditioning regimen through the entire posttransplant period.

All Polish hematopoietic cell transplant centers were invited to participate in the survey on proposed prophylactic pharmacological approach for patients undergoing auto- or allo-HCT, both in pediatric and adult settings. This report presents the results of the survey.

\section{Methods}

During Transplant Workshop of Polish Federation of Bone Marrow Transplant Centers in Poznan, dated October 12, 2019, a survey on proposed antimicrobial prophylaxis was distributed, and one questionnaire per center was prepared. Based on their own experience, the centers were asked to suggest antibacterial, antifungal, and antiviral prophylaxis in auto- and alloHCT settings, with respect to the pretransplant phase, neutropenic phase, immunosuppressive prophylaxis phase and in case of GVHD. Additional questions were related to other supportive therapies and key diagnostic tests. Both pediatric and adult centers answered the same questionnaire. Vaccinations were not included in the survey.
Article history:

Received: 01.03.2020

Accepted: 22.03.2020

Jan Styczyński ${ }^{1,{ }^{*},}$, Krzysztof Czyżewski', Marek Ussowicz², Grzegorz Helbig ${ }^{3}$, Małgorzata Sobczyk-Kruszelnicka ${ }^{4}$, Anna Łojko ${ }^{5}$, Anna Czyż ${ }^{6}$, Beata Piątkowska-Jakubas ${ }^{7}$, Agnieszka Sobkowiak-Sobierajska ${ }^{8}$, Agnieszka Zaucha-Prażmo ${ }^{9}$, Aleksandra Krasowska-Kwiecień ${ }^{10,11}$, Kazimierz Hałaburda ${ }^{12}$, Piotr Boguradzki ${ }^{13}$ Maria Bieniaszewska ${ }^{14}$, Agnieszka Wierzbowska ${ }^{15}$, Piotr Rzepecki ${ }^{16}$, Adam Walter-Croneck ${ }^{17}$, Mariola Sędzimirska ${ }^{18}$, Agnieszka Druzd-Sitek ${ }^{19}$, Dorota Hawrylecka ${ }^{20}$, Bartłomiej Baumert ${ }^{21}$, Ewa Lutwin ${ }^{22}$, Edyta Cichocka ${ }^{23}$ Katarzyna Smalisz ${ }^{24}$, Lidia Gil

Department of Pediatric Hematology and Oncology, Collegium Medicum, Nicolaus Copernicus University Torun, Bydgoszzz, Poland Department of Pediatric Transplantology peparment of Wroctogy and Oncology, Medical University, Wroctaw, Poland

Department of Hematology, Medical University of Silesia, Katowice, Poland

Department of Bone Marrow Transplantation and Oncohematology Maria Sklodowska-Cure National Pesearch instive of Gearch Institute of Oncology Gliwice Branch Gliwice, Poland

Department of Hematology, Poznan University of Medical Sciences, Poznań, Poland Department of Hematology, Medical University, Wroctaw Poland

Department of Hematology, Collegium Medicum, Jagiellonian University, Kraków, Poland ${ }^{8}$ Department of Pediatric Hematology, Oncology and Transplantology, Medical University, Poznar Poland

Department of Pediatric Hematology Oncology 'Department of Pediatric Hematology, Oncology and Transp

Polan

${ }^{10}$ Department of Transplantation, Children's University Hospital, Kraków, Poland "Department of Clinical Immunology and Transplantology _Lagiellonian University Medica College, Kraków, Poland "Department of Hematology, Institute of Hematology and Transfusion Medicine, Warszawa, Poland

${ }^{3}$ Department of Hematology, Oncology and Internal Medicine, Medical University, Warsaw Poland

Department of Hematology, Medical University, Gdan'sk, Poland

${ }^{15}$ Department of Hematology, Medical University, tódż, Poland

${ }^{16}$ Department of Hematology, Military Institute of Medicine, Warsaw, Poland

Tone Marrow ransplantation, Medical University, Lublin, Poland 'Lower Silesian Center for Cellular Transplantation and National Bone Marrow Donor Registry, Wroctaw, Poland

19Department of Lymphoid Malignancies, Maria Skepartmentofly to Oncorgy Warsawa Poland Oncology, Warszawa, Poland ${ }^{20}$ Departament of Oncological Hematology and Transplantology, Podkarpacie Oncological Center, Brzozów, Poland

"Department of Hematology and Transplantology Medical University Szczecin Podand

Medical Unversity, Szczecin, Poland Department of Mematology and Transplantology, enter of Oncology, Kielce, Poland ${ }^{2}$ Department of Hematology, Nicolaus Copernicus Town Hospital, Toruń, Poland ${ }^{24}$ Department of Pediatric Hematology and Oncology and Clinical ansplantogy Oncology and Clinical Transplant

${ }^{*}$ Corresponding author: Jan Styczyński, Department of Pediatric Hematology and Oncology, Collegium Medicum, Nicolaus Copernicus University Toruń, Skłodowskiej-Curie 9, 85-094 Bydgoszcz, Poland, phone: +48 52 5854860, fax: +4852 5854087, e-mail: jstyczynski@cm.umk.pl 
Five adult and one pediatric centers perform auto-HCT only; however, they partially answered the allo-HCT section, as the survey questions were about the recommended approach.

No Bioethical Committee agreement was necessary for this study, as no patients were involved. All participants of the survey are coauthors of this manuscript and they gave their agreement for publication.

\section{Results and Discussion}

A total number of 23 centers participated, including 6 for pediatric and 17 for adult patients. The summary of prophylaxis proposed by the centers with respect to auto- and allo-HCT and with respect to pre- and posttransplant phases in allo-HCT setting is shown in table I.

\section{Prophylaxis in auto- and allo-HCT settings}

\section{Antibacterial prophylaxis}

More than half of adult centers both in auto-and allo-HCT patients prefer to use quinolones (ciprofloxacin or levofloxacin). Some of the adult centers are in favor of use of oral penicillins (amoxicillin \pm clavulanate or V-cyllin) mainly in allo-HCT setting. The choice of prophylaxis in pediatric centers is split between ciprofloxacin and amoxicillin, both in allo- and auto-HCT settings. Additionally, half of the centers are willing to use V-cyllin after allo-HCT. The prophylactic use of other antibacterial compounds is rare both in pediatric and adult centers.

\section{Antifungal prophylaxis}

All pediatric and adult centers use fluconazole in patients after auto$\mathrm{HCT}$, and selection of antifungals in allo-HCT patients is variable and it includes posaconazole, voriconazole, fluconazole, and micafungin, with the exception of general avoidance of prophylactic use of voriconazole in pediatric centers. One adult center uses caspofungin during conditioning before allo-HCT with antithymocyte globulin (ATG). More than half adult centers perform regular weekly testing for galactomannan (GM) in all allo- and auto-HCT patients. It is also the rule for allo-HCT, but not for auto-HCT pediatric patients.

\section{Antiviral prophylaxis}

All adult centers use acyclovir in allo- and auto-HCT settings, and all pediatric centers use acyclovir in allo-HCT patients while only exceptionally in auto-HCT setting. One adult center uses ganciclovir during conditioning before allo-HCT with ATG. Almost all adult and pediatric centers perform regular weekly testing for Cytomegalovirus (CMV) DNA and Epstein-Barr virus (EBV) DNA in allo-HCT patients. Some adult centers emphasize the necessity of use of letermovir in CMV-seropositive recipients.

\section{Other antimicrobial prophylaxis}

Almost all centers use cotrimoxazole in prophylaxis against pneumocystodosis and toxoplasmosis. Almost all pediatric and a few adult centers perform regular intravenous immunoglobulin (IVIG) supplementation, mainly in allo-HCT patients.

\section{Prophylaxis in various phases of allo-HCT}

\section{Antibacterial prophylaxis}

Antibacterial prophylaxis in allo-HCT setting is rarely used during conditioning period by the centers, while virtually all pediatric and adult centers use prophylaxis both during neutropenia and immunosuppressive treatment and/or GVHD phase.

\section{Antifungal prophylaxis}

Antifungal prophylaxis in allo-HCT setting is very rarely used by adult centers during conditioning period, while virtually all pediatric and adult centers use antifungal prophylaxis both during neutropenia and immunosuppressive treatment and/or GVHD phase. Additionally, more than half pediatric centers also use antifungal prophylaxis during conditioning. None of the centers use voriconazole during conditioning. Itraconazole tablets are used exceptionally in one center only.

\section{Antiviral prophylaxis}

Acyclovir is recommended for prophylaxis both before HCT and during neutropenia and immunosuppressive treatment by almost all centers.

\section{Other antimicrobial prophylaxis}

Cotrimoxazole in prophylaxis is used both during neutropenia and immunosuppressive therapy periods in almost all centers, and also in part of the centers before HCT. Supplementation of IVIG to maintain concentration $4 \mathrm{~g} / \mathrm{L}$ is performed both during neutropenia and immunosuppressive therapy in almost all centers.

\section{Clinical implications of the survey}

The results of this survey underline the value of antimicrobial prophylaxis in patients undergoing HCT and good awareness of this issue in all transplant centers. Virtually all pediatric and adult centers use antibacterial, antifungal, antiviral, and anti-pneumocystodosis prophylaxis both in allo- and auto-HCT patients. There are some differences regarding specific drugs, especially with respect to the lower use of quinolones in pediatric patients. The most preferred combinations of drugs are: quinolones and penicillins, fluconazole and posaconazole, and acyclovir and cotrimoxazole. Posaconazole and voriconazole are used exclusively in allo-HCT patients in primary and secondary prophylaxis as a result of reimbursement program of these drugs in Poland. This is also in line with international guidelines of antimicrobial prophylaxis.

The results of this survey reflect pharmacological antimicrobial prophylaxis practices used in Polish transplant centers. They also reflect drug availability and reimbursement in Poland, since letermovir, a new anti-CMV compound, recommended for CMVseropositive allo-HCT recipients is not used in Poland.

This survey may be used to create universal national guidelines for Polish HCT centers which are compatible with international guidelines 
Table I. Proposed anti-infective prophylaxis

\begin{tabular}{|c|c|c|c|c|c|c|c|c|c|}
\hline & \multicolumn{2}{|c|}{ Pediatric $(n=6)$} & & \multicolumn{2}{|c|}{ Adult $(n=17)$} & & \multicolumn{3}{|c|}{ Total $(n=23)$} \\
\hline & Auto & & Allo & Auto & \multicolumn{2}{|c|}{ Allo } & Auto & \multicolumn{2}{|r|}{ Allo } \\
\hline \multicolumn{10}{|l|}{ Antibacterial } \\
\hline Quinolones: Ciprofloxacin & \multicolumn{2}{|l|}{2} & 4 & 9 & \multicolumn{2}{|c|}{8} & 11 & \multicolumn{2}{|r|}{12} \\
\hline Levofloxacin & - & & - & 6 & 6 & & 6 & & 6 \\
\hline Penicillins: Amoxicillin \pm Clavulanian & 3 & & 4 & 1 & 5 & & 4 & & 9 \\
\hline V-cyllin & - & & 3 & 1 & 8 & & 1 & & 11 \\
\hline Cephalosporines: Cefuroxime & 1 & & 1 & 1 & - & & 2 & & 1 \\
\hline Aminoglycosides (oral) & 1 & & 1 & - & - & & 1 & & 1 \\
\hline Macrolides: Azithromycin & - & & 1 & - & - & & - & & 1 \\
\hline Rifaximin & - & & - & 1 & 1 & & 1 & & 1 \\
\hline Antifungal & & & & & & & & & \\
\hline Posaconazole & - & & 4 & - & 12 & & - & & 16 \\
\hline Voriconazole & - & & 1 & - & 10 & & - & & 11 \\
\hline Fluconazole & 6 & & 5 & 17 & 10 & & 23 & & 15 \\
\hline Itraconazole & - & & 1 & 1 & 1 & & 1 & & 2 \\
\hline Mycamine & - & & 3 & - & 7 & & - & & 10 \\
\hline Galactomannan testing $\geq 1 /$ week & 1 & & 4 & 8 & 12 & & 9 & & 16 \\
\hline Other: Mannan or BDG & - & & - & 2 & 2 & & 2 & & 2 \\
\hline Antiviral & & & & & & & & & \\
\hline Acyclovir & 1 & & 6 & 17 & 16 & & 18 & & 22 \\
\hline Other & - & & - & - & - & & - & & - \\
\hline CMV DNA $\geq 1 /$ week & - & & 6 & - & 14 & & - & & 20 \\
\hline EBV DNA $\geq 1 /$ week & - & & 6 & - & 12 & & - & & 18 \\
\hline Other antimicrobial prophylaxis & & & & & & & & & \\
\hline Cotrimoxazole & 4 & & 6 & 17 & 13 & & 21 & & 19 \\
\hline IVIG supplementation & 3 & & 5 & - & 6 & & 3 & & 11 \\
\hline Other: pentamidine & 1 & & 1 & - & - & & 1 & & 1 \\
\hline Prophylaxis in allo-HCT patients wi & respect to & ransplant phas & & & & & & & \\
\hline & Pediatric ( & $=6)$ & & Adult $(\mathrm{n}=$ & & & Total $(\mathbf{n}=$ & & \\
\hline & Pre HCT & Neutropenia & GVHD IST & Pre HCT & Neutropenia & GVHD IST & Pre HCT & Neutropenia & GVHD IST \\
\hline Antibacterial & & & & & & & & & \\
\hline Quinolones: Ciprofloxacin & 2 & 4 & 4 & 1 & 8 & 6 & 3 & 12 & 10 \\
\hline Levofloxacin & - & - & - & 3 & 7 & 7 & 3 & 7 & 7 \\
\hline Penicillins: Amoxicillin \pm Clavulanian & 1 & 4 & 4 & 1 & 2 & 6 & 2 & 6 & 10 \\
\hline V-cyllin & - & 1 & 4 & - & 1 & 8 & - & 2 & 12 \\
\hline Cephalosporines: Cefuroxime & - & - & - & - & 1 & - & - & 2 & - \\
\hline Aminoglycosides (oral) & 1 & - & - & - & - & - & 1 & - & - \\
\hline Macrolides: Azithromycin & - & 1 & 1 & - & - & - & - & 1 & 1 \\
\hline Rifaximin & - & - & - & - & 1 & - & - & 1 & - \\
\hline Antifungal & & & & & & & & & \\
\hline Posaconazole & 3 & 3 & 4 & 1 & 3 & 12 & 4 & 6 & 16 \\
\hline Voriconazole & - & 1 & 1 & - & 5 & 9 & - & 6 & 10 \\
\hline Fluconazole & 4 & 5 & 4 & 4 & 15 & 6 & 8 & 20 & 10 \\
\hline Itraconazole & - & - & 1 & - & - & - & - & - & 1 \\
\hline Mycamine & 2 & 2 & 2 & 1 & 5 & 2 & 3 & 7 & 5 \\
\hline Galactomannan testing $\geq 1 /$ week & 1 & 4 & 4 & 3 & 10 & 8 & 4 & 14 & 12 \\
\hline Other: Mannan or BDG & - & - & - & - & 2 & 1 & - & 2 & 1 \\
\hline Antiviral & & & & & & & & & \\
\hline Acyclovir & 3 & 6 & 6 & 13 & 15 & 14 & 16 & 21 & 20 \\
\hline Other & - & - & - & - & - & - & - & - & - \\
\hline CMV DNA $\geq 1 /$ week & 4 & 6 & 6 & 2 & 7 & 11 & 6 & 13 & 17 \\
\hline EBV DNA $\geq 1 /$ week & 4 & 6 & 6 & - & 5 & 10 & 4 & 11 & 16 \\
\hline Other antimicrobial prophylaxis & & & & & & & & & \\
\hline Cotrimoxazole & 4 & 5 & 6 & 7 & 11 & 11 & 11 & 16 & 17 \\
\hline IVIG supplementation & 1 & 5 & 5 & 1 & 2 & 6 & 2 & 7 & 11 \\
\hline Other: pentamidine & 1 & 1 & 1 & - & - & - & 1 & 1 & 1 \\
\hline
\end{tabular}

BDG - beta-d-glucan; GVHD - graft-versus-host disease; IST - immunosuppressive therapy; IVIG - intravenous immunoglobulin 
[7-15]. It may also serve as a useful tool for any local or multicenter epidemiological analyses.

\section{Authors' contributions}

JS, LG - involved in design of the study. JS, LG - wrote manuscript. JS, LG, A, , KC - performed survey. MU, AC, LG, KC - involved in critical review. All authors approved the final manuscript.

\section{Conflict of interest}

All authors have nothing to disclose with respect to this paper.

\section{Financial support}

None.

\section{Ethics}

The work described in this article has been carried out in accordance with the Code of Ethics of the World Medical Association (Declaration of Helsinki) for experiments involving humans; EU Directive 2010/63/ EU for animal experiments; Uniform requirements for manuscripts submitted to biomedical journals.

\section{References}

[1] Styczynski J, Tridello G, Koster L, et al. Death after hematopoietic stem cell transplantation: changes over calendar year time, infections and associated factors. Bone Marrow Transplant 2020;55:126-36.

[2] Styczynski J, Czyzewski K, Wysocki M, et al. Increased risk of infections and infection-related mortality in children undergoing haematopoietic stem cell transplantation compared to conventional anticancer therapy: a multicentre nationwide study. Clin Microbiol Infect 2016;22:179.e1-e10.

[3] Czyzewski K, Styczynski J, Giebel S, et al. Age-dependent determinants of infectious complications profile in children and adults after hematopoietic cell transplantation: lesson from the nationwide study. Ann Hematol 2019;98(9):2197-211.

[4] Czyzewski K, Galazka P, Fraczkiewicz J, et al. Epidemiology and outcome of invasive fungal disease in children after hematopoietic cell transplantation or treated for malignancy: Impact of national programme of antifungal prophylaxis. Mycoses 2019;62:990-8.

[5] Styczynski J. Infectious complications in children and adults with hematological malignancies. Acta Haematol Pol 2019;50:167-73.

[6] Styczynski J. ABC of viral infections in hematology: focus on herpesviruses. Acta Haematol Pol 2019;50:159-66.

[7] Styczynski J, van der Velden W, Fox CP, et al. Management of EpsteinBarr Virus infections and post-transplant lymphoproliferative disorders in patients after allogeneic hematopoietic stem cell transplantation: Sixth European Conference on Infections in Leukemia (ECIL-6) guidelines. Haematologica 2016;101:803-11.

[8] Matthes-Martin S, Feuchtinger T, Shaw PJ, et al. European guidelines for diagnosis and treatment of adenovirus infection in leukemia and stem cell transplantation: summary of ECIL-4 (2011). Transpl Infect Dis 2012;14:555-63.

[9] Ljungman P, de la Camara R, Robin C, et al. Guidelines for the management of cytomegalovirus infection in patients with haematological malignancies and after stem cell transplantation from the 2017 European Conference on Infections in Leukaemia (ECIL 7). Lancet Infect Dis 2019;19:e260-72.
[10] Engelhard D, Mohty B, de la Camara R, Cordonnier C, Ljungman P. European guidelines for prevention and management of influenza in hematopoietic stem cell transplantation and leukemia patients: summary of ECIL-4 (2011), on behalf of ECIL, a joint venture of EBMT, EORTC, ICHS, and ELN. Transpl Infect Dis 2013;15:219-32.

[11] Averbuch D, Orasch C, Cordonnier C, et al. European guidelines for empirical antibacterial therapy for febrile neutropenic patients in the era of growing resistance: summary of the 2011 4th European Conference on Infections in Leukemia. Haematologica 2013;98:1826-35.

[12] Groll AH, Castagnola E, Cesaro S, et al. Fourth European Conference on Infections in Leukaemia (ECIL-4): guidelines for diagnosis, prevention, and treatment of invasive fungal diseases in paediatric patients with cancer or allogeneic haemopoietic stem-cell transplantation. Lancet Oncol 2014;15:e327-40.

[13] Tissot F, Agrawal S, Pagano L, et al. ECIL-6 guidelines for the treatment of invasive candidiasis, aspergillosis and mucormycosis in leukemia and hematopoietic stem cell transplant patients. Haematologica 2017;102:433-44.

[14] Maertens J, Cesaro S, Maschmeyer G, et al. ECIL guidelines for preventing Pneumocystis jirovecii pneumonia in patients with haematological malignancies and stem cell transplant recipients. J Antimicrob Chemother 2016;71:2397-404.

[15] Maertens J, Marchetti O, Herbrecht R, et al. European guidelines for antifungal management in leukemia and hematopoietic stem cell transplant recipients: summary of the ECIL $3-2009$ update. Bone Marrow Transplant 2011;46:709-18. 